\title{
Hydroxyurea stärkt Lunge bei Sichelzellanämie
}

\author{
Hydroxyurea wird bei Sichelzellanämie bisher eher selten eingesetzt - \\ wohl zu Unrecht, wie auf dem ATS-Kongress 2016 berichtet wurde.
}

Patienten mit Sichelzellanämie verlieren ab der frühen Kindheit Jahr für Jahr an Lungenfunktion. Die zytostatisch wirksame Hydroxyurea bessert neben anderen Krankheitssymptomen anscheinend auch den Lungenfunktionsverlust.

Das geht aus einer kanadischen Studie an 94 jungen Sichelzell-Patienten zwischen 6 und 20 Jahren hervor, die fast vier Jahre lang offen mit Hydroxyurea behandelt wurden. Spirometrische Daten liegen aus den vier Jahren sowie von sieben Zeitpunkten während der Studie (3, 6, 9, 12, 24, 26 und 48 Monate) vor.

Unter der Therapie verlangsamte sich der Lungenfunktionsrückgang um mehr als ein Drittel von $2 \%\left(\mathrm{FEV}_{1}\right)$ respektive Krankheitsdauer.

\section{Sorge vor Nebenwirkungen} nogenesen kommt.

$3,6 \%$ (FEF25-75\%) pro Jahr auf 1,3\% und $2,9 \%$ pro Jahr $(\mathrm{p}<0,05)$, berichtete Studienleiterin Dr. Anya McLaren, Kinderkrankenhaus Toronto. Die Wirkung zeigte sich unabhängig von Alter oder

Hydroxyurea wird bei Sichelzellanämie aufgrund von Toxizitätsbedenken bisher eher selten eingesetzt, so McLaren. Aber diese Bedenken seien unbegründet: „Langzeitbeobachtungen deuten auf günstige Effekte hin, ohne das es zu Knochenmarksschäden, Wachstumsverzögerungen, Fertilitätsstörungen oder Karzi-

Manuela Arand

\section{Prävention im Kleinkindalter}

\section{Allergene Beikost? Je früher, desto besser!}

\section{Das Allergierisiko scheint bei Kindern geringer zu sein, wenn sie früh mit allergenen Nahrungsmitteln in Berührung kommen.}

Kinder, die im ersten Lebenshalbjahr nur gestillt werden, entwickeln später häufiger allergische Sensibilisierungen gegen Nahrungsbestandteile als die, die früh Beikost mit potenten Allergenen bekommen haben. Das ergab eine Subanalyse der CHILD („Canadian Healthy Infant Longitudinal Development")-Studie, in die Daten von über 1.400 Kindern einer Geburtskohorte eingegangen sind. Die Studie untersuchte, wie der Zeitpunkt, an dem Umweltfaktoren auf ein Kind einwirken, das spätere Risiko für Asthma und Allergien beeinflusst.

Für die Substudie wurden drei Nahrungsmittel ausgewählt, die als besonders allergen gelten: Kuhmilch, Erdnuss und Hühnerei. Es zeigte sich, dass das Sensibilisierungsrisiko umso geringer ausfiel, je früher die Kinder diese Nahrungsmittel erhielten, berichtete Maxwell Tran, Forschungsstudent an der McMaster University, Hamilton. Ganz besonders deutlich wurde das beim Hühnerei. Kinder, die schon im ersten Lebensjahr eihal- tige Beikost erhalten hatten, entwickelten wesentlich seltener eine Sensibilisierung gegen eines der drei Nahrungsmittel (adjustierte Odds Ratio 0,33 bei Zufütterung in den ersten 6 Monaten, 0,47 bei Zufütterung in Monat 7 bis 12).

Entgegen der landläufigen Meinung waren Kinder, die im ersten Halbjahr nur Muttermilch bekommen hatten, nicht besser geschützt - im Gegenteil: Sie entwickelten öfter eine Sensibilisierung gegen Kuhmilch. „Sensibilisierung ist nicht Allergie“, betonte Tran. „Aber sie ist ein wichtiger Schritt auf dem Weg dorthin." (ara)

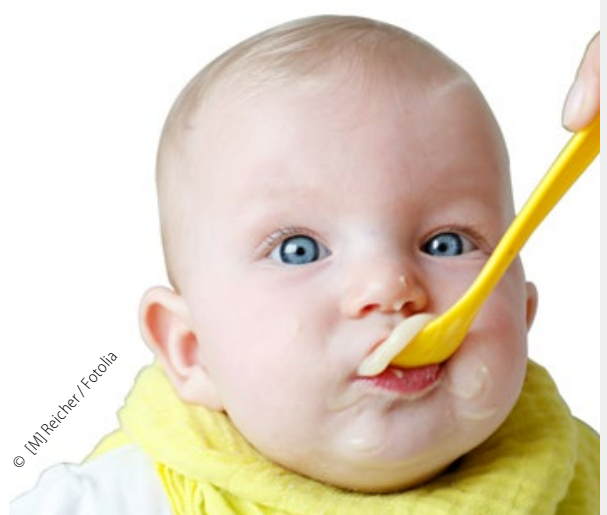

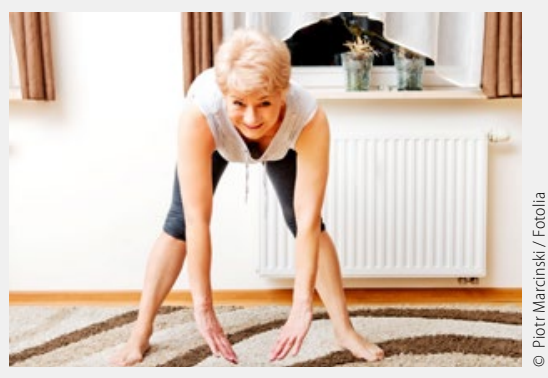

\section{Lungen-Reha funktioniert auch zuhause}

COPD-Patienten können eine Lungen-Rehabilitation auch erfolgreich zuhause durchführen, berichteten Autoren um Prof. Anne Holland, Alfred Health and La Trobe University, Melbourne.

Obgleich Lungen-Reha den Patienten vor Exazerbationen schützt, die Symptome lindert und die Belastbarkeit erhöht, nehmen nur etwa $10 \%$ der COPD-Patienten die Chance wahr. Neben der Motivation fehlt es häufig an LungenReha-Plätzen in der Umgebung.

Die Autoren hatten daher die klassische ambulante Lungen-Reha mit einer Reha zuhause über acht Wochen verglichen, wobei das ambulante Programm einen anfänglichen Besuch eines Physiotherapeuten sowie wöchentliche Motivations-Anrufe vorsah. Die Kontrollgruppe nahm zweimal wöchentlich an Trainingseinheiten teil.

Sowohl nach acht Wochen als auch nach einem Jahr schnitten beide Gruppen gleich ab in Bezug auf Belastbarkeit, Symptomkontrolle und Selbstbewusstsein.

\section{Kognitive Defizite bei schnarchenden Kindern}

Kinder mit Atemstörungen im Schlaf aufgrund habituellen Schnarchens oder vergrößerter Mandeln haben mehr Schwierigkeiten in der Schule, berichteten Autoren einer großen pädiatrischen Studie. Sie stellten bereits messbare kognitive Defizite fest bei Kindern, die lediglich schnarchten.

Für ihre Studie rekrutierten die Forscher um Dr. David Gozal, University of Chicago, 1.359 Kinder im Alter von 5 bis 7 Jahren. Die Untersuchungen beinhalteten eine Erhebung des Schlafes mittels Fragebogen, eine nächtliche Polysomnografie sowie ausführliche neurokognitive Tests.

Die Kinder wurden in vier Gruppen eingeteilt (1: Nicht-Schnarcher; 2: Schnarcher; 3: Schnarcher mit leichter Apnoe; 4: Schnarcher mit deutlicherer Apnoe).

Die Ergebnisse zeigten, dass die Kinder mit zunehmender Schwere der nächtlichen Atemprobleme mehr kognitive Probleme offenbarten. Die Defizite betrafen beispielsweise die Aufmerksamkeit, das Gedächtnis und die Sprache. Die Autoren empfehlen dringend, schnarchende Kinder kognitiv abzuklären und früh zu intervenieren. 